\title{
Los corredores urbanos Periférico Sur e Insurgentes Sur y su impacto en el patrimonio cultural edificado de la Ciudad de México (1980-2018)
}

Urban Corridors Periferico Sur and Insurgentes Sur and its Impact on Mexico City Cultural Built Heritage (1980-2018)

\author{
Israel Román Ramos*
}

Recibido: 20 de junio de 2018

Aceptado: 20 de diciembre de 2018

\section{Resumen}

En este documento se examina, desde una perspectiva histórica, cómo es que las políticas neoliberales se tornan las principales causas de afectación al patrimonio cultural edificado hacia la porción sur de la Ciudad de México. En primer lugar, se recapitula el desarrollo del patrimonio cultural edificado en México durante el siglo XX. Se subraya el hecho que esta noción se acompaña también de una serie de procesos destructivos en torno al mismo. La trayectoria continúa con las transformaciones morfológicas y funcionales ocurridas en los corredores urbanos Periférico Sur e Insurgentes Sur, presentando información de carácter cuantitativo en relación con la concentración de complejos de edificaciones y usos de suelo, que se dedican al sector terciario en la actualidad. Se subraya una relación actual entre las políticas neoliberales y las contradicciones en la preservación de bienes patrimoniales a nivel local. El primer caso es la Zona arqueológica de Cuicuilco y su falta de mantenimiento por reducciones al gasto público; el segundo es la reconversión de una antigua fábrica en centro comercial mediante la participación de capital privado; el tercero son las esculturas monumentales que permanecieron abandonadas por sus propietarios y carecieron de protección oficial por las instituciones mexicanas durante años.

Palabras clave: corredor urbano, patrimonio cultural edificado, políticas neoliberales, sector terciario.

\begin{abstract}
In this paper we examine, from a historical perspective, how neoliberal policies become the underlying causes that affect cultural built heritage in the southern portion of Mexico City, at present. First, we document the development of cultural built heritage in Mexico during the 20th century. We underline that this idea is accompanied, however, by a series of destruction processes concerning cultural built heritage at the same time, as the urban sprawl of Mexico City expands far south. Then, we focus on morphological and functional transformations that occurred in Periferico Sur and Insurgentes Sur urban corridors. Quantitative data that support an amalgamation of building complexes and land uses is presented, which is strongly related to the tertiary sector. We highlight the contradictions concerning the preservation of cultural built heritage within the region in current days. A first case study deals with Cuicuilco Archaeological Zone and the lack of public investment in its maintenance. Second, we assess the reconversion of an old factory into a shopping mall with support of private investment. Finally, we deal with a group of monumental sculptures that remained abandoned by their owners, but also lack of any kind of official protection by the Mexican institutions, for years.
\end{abstract}

Keywords: cultural built heritage, neo-liberal policies, tertiary sector, urban corridor.

\footnotetext{
* Filiación: Universidad Nacional Autónoma de México, México. Contacto: isra_roman@ @otmail.com
}

Cómo citar: Román Ramos, I. (2018). Los corredores urbanos Periférico Sur e Insurgentes Sur y su impacto en el patrimonio cultural edificado de la Ciudad de México (1980-2018). Revista de Urbanismo, 39, 1-18. https://doi.org/10.5354/0717- 


\section{Introducción}

El objetivo general de la presente investigación es demostrar que las políticas neoliberales son las causas estructurales de afectación y destrucción al patrimonio cultural edificado, que actualmente prevalece en dos de los corredores urbanos más importantes localizados hacia el suroeste de la Zona Metropolitana del Valle de México [ZMVM]: el Anillo Periférico Boulevard Adolfo Ruiz Cortines (Periférico Sur) y la Avenida Insurgentes Sur. Para lograr dicho cometido, la hipótesis citada a continuación sirve de hilo conductor en todo el análisis: La implementación de políticas neoliberales en México ha generado (in)acciones hacia el patrimonio cultural edificado presente en los corredores urbanos Periférico Sur e Insurgentes Sur, ya que sólo obedecen a los (des)intereses de la dinámica de acumulación de capital privado, y pueden culminar en la incapacidad del Estado de garantizar la preservación de este.

El impacto del capitalismo en las políticas de las ciudades ha sido objeto de múltiples trabajos críticos (Borja, 2007; Smith, 1984), incluyendo las áreas patrimoniales o los centros históricos (Carrión, 2012). Para los fines de esta investigación se retoma la definición de políticas neoliberales de Harvey (2007, p. 21), quien refiere a una "teoría de prácticas políticas económicas" fundamentadas en el supuesto alcance del "bienestar humano a través de otorgar grandes libertades empresariales dentro de un marco institucional creado por el Estado". A lo largo de la investigación se brinda especial atención a las políticas que favorecen la desregularización de bienes y servicios del Estado, el otorgamiento de derechos de propiedad privada en zonas que inicialmente pertenecen al Estado, y los ajustes fiscales basados en la reducción al gasto público. Desde la postura de este trabajo, estas prácticas políticas económicas están lejos de atender y solucionar los problemas inherentes a la recuperación y preservación del patrimonio cultural edificado presente en la zona sur de la Ciudad de México, puesto que en la práctica sólo se focalizan en la reproducción de capital.

La presente problemática deriva del hecho que los corredores urbanos Periférico Sur e Insurgentes Sur han atravesado por una rápida transformación morfológica y funcional como ejes estructuradores de la ZMVM, a partir del último tercio del siglo XX. Por una parte, ocurre una reestructuración urbana en cuanto a forma y función de ambos ejes, inclinada al desarrollo del sector terciario mediante una fuerte participación del sector privado. Por otra parte, existe un importante conjunto de monumentos muebles e inmuebles a lo largo de ambos corredores urbanos, los cuales son valorados como patrimonio cultural edificado por las leyes mexicanas y los tratados internacionales gracias a sus valores históricos, estéticos, académicos, sociales y simbólicos.

El trabajo se desarrolla desde un enfoque histórico subdividido en tres apartados; es decir, a manera de una línea del tiempo dentro de la zona sur de la Ciudad de México, enriqueciendo la discusión con algunos factores de carácter económico y político que van desarrollándose en este contexto gradualmente. Por ende, en el primer apartado se examinan los orígenes del concepto de patrimonio cultural edificado en México. Se presentan los fundamentos de la institucionalización del patrimonio nacional que se remontan a principios del siglo $\mathrm{XX}$, los cuales se conjugan a la postre con los tratados internacionales relativos al tema. De tal forma, el análisis decanta en un proceso de valoración de ciertas áreas y monumentos en la zona sur de la Ciudad de México que culmina en la década de 1980, mismos que eventualmente quedarán dispersos a lo largo de los corredores urbanos Periférico Sur e Insurgentes Sur.

En el segundo apartado se aborda la dinámica territorial de los corredores urbanos Periférico Sur e Insurgentes Sur en un lapso que va de 1980 al 2016, brindando especial atención al sector terciario durante el último año. Específicamente, el corredor urbano Periférico Sur queda delimitado por el tramo intermedio a la Glorieta de San Jerónimo y la entrada al embarcadero de Cuemanco Xochimilco. Por su parte, el corredor urbano Insurgentes Sur comprende desde la Avenida de la Paz hasta la calle Calvario (Figura 1). En lo que concierne a la metodología de análisis inherente al sector terciario, ésta es de carácter cuantitativo y espacial. El principal soporte es la identificación y cuantificación de servicios avanzados dentro de ambos corredores urbanos, valiéndose de una tabla general que engloba diez categorías de análisis ligadas al sector terciario que provienen, en su mayoría, de los trabajos de Taylor (2004) y Hall (2001). Acto seguido, se identifican geográficamente y contabilizan las unidades económicas que presenta el Directorio Estadístico Nacional de Unidades Económicas [DENUE] del 
INEGI (2016) dentro de los corredores urbanos Periférico Sur e Insurgentes Sur, en orden de dilucidar la variabilidad y cantidad de empresas/firmas que se dedican al sector terciario en esta región. En el tercer apartado se analiza cómo impacta la reestructuración morfológica y funcional de Periférico Sur e Insurgentes Sur en el patrimonio cultural edificado.

La lista de monumentos patrimoniales en los corredores urbanos Periférico Sur e Insurgentes Sur es amplia y corresponde a diferentes periodos de la historia de México. Destacan la Zona arqueológica de Cuicuilco, el Cerro Zacatépetl, al igual que los canales y las chinampas de Xochimilco, cuyo origen se remonta a la época prehispánica. Asimismo, la capilla El Calvario y el exconvento del Carmen tienen relación con los pueblos periféricos a la Ciudad de México refundados por los españoles durante el periodo colonial (siglos XVI-XIX). Igualmente, sobresalen la ex-fábrica de Peña Pobre y el Parque de la Bombilla por su correspondencia a la segunda mitad del siglo XIX y primer tercio del siglo XX (inicios del México independiente y México posrevolucionario). Por último, el Campus Central de Ciudad Universitaria y las esculturas conmemorativas de la XIX Olimpiada celebrada en 1968 se insertan en la historia del México contemporáneo (segunda mitad del siglo XX hasta el presente).

Ante la pluralidad de bienes patrimoniales en esta región, el trabajo se focaliza en tres casos de monumentos que subsisten en ambos corredores urbanos. Dicha selección obedece a los siguientes criterios: 1) la temporalidad en concordancia con los distintos periodos de la historia mexicana; 2) el régimen de propiedad (privada o federal); 3) la procedencia de los recursos destinados a ellos (recursos públicos o capital privado); y 4) los tipos de intervención realizados en ellos (apertura al público, rescate, reconversión, restauración o reubicación).

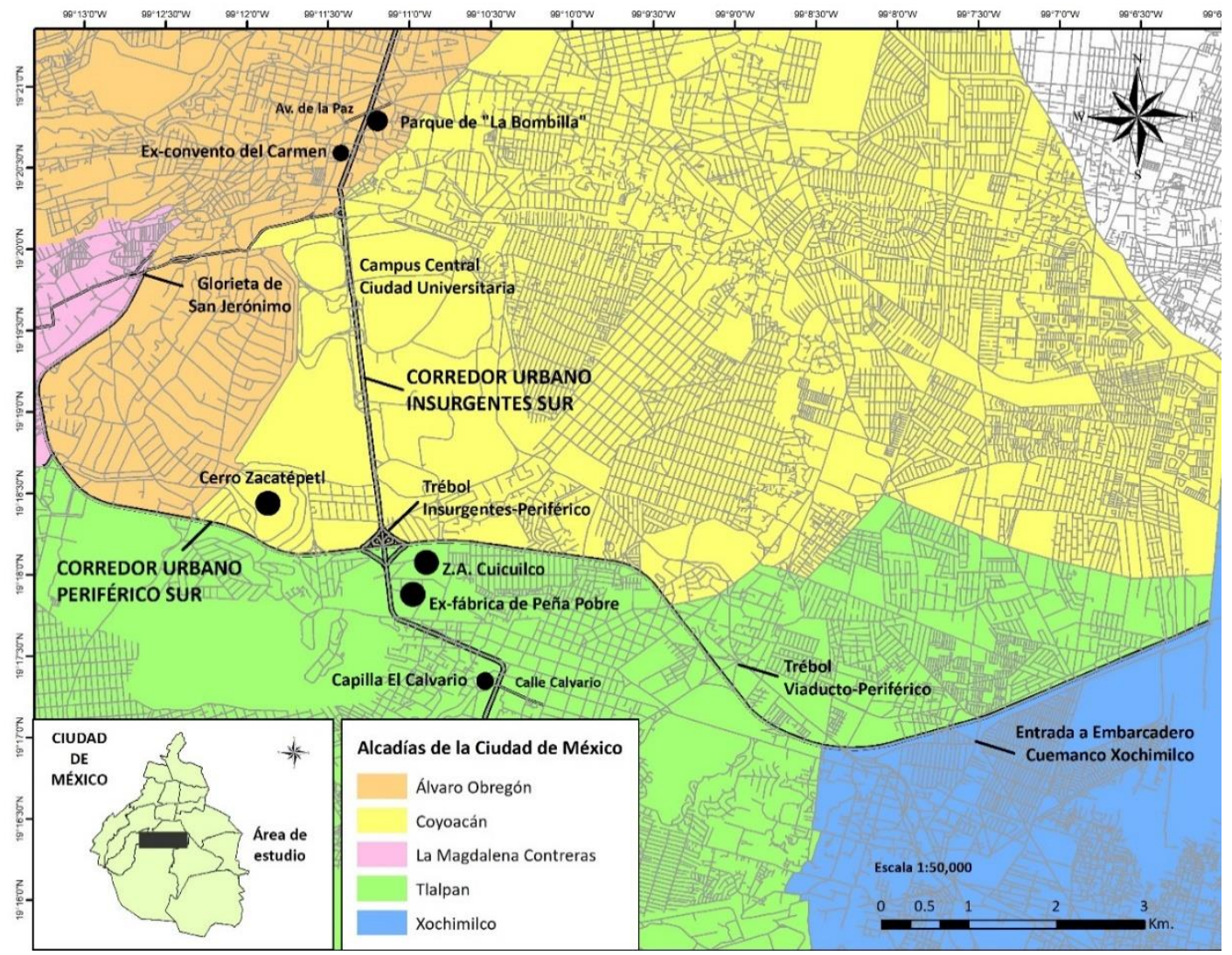

Figura 1. Ubicación de los corredores urbanos Periférico Sur e Insurgentes Sur hacia la porción suroeste de la Ciudad de México.

Fuente: Elaboración propia basada en cartografía digital de INEGI (2015). (Sistema de Coordenadas Geográficas Datum ITRF_1992). 
De tal suerte, el primer caso de análisis es la Zona arqueológica de Cuicuilco: un área de propiedad federal cuyo origen se remonta a la época prehispánica; se encuentra abierta al público de forma gratuita y se sostiene de recursos públicos asignados al Instituto Nacional de Antropología e Historia [INAH] por su carácter de dependencia federal. El segundo caso de análisis es la ex-fábrica de Peña Pobre: un edificio del siglo XIX de propiedad privada; fue reconvertido en una plaza comercial y en la sede principal de dos corporativos mediante la inversión de capital privado. Por último, el tercer caso de análisis son las esculturas conmemorativas de la XIX Olimpiada celebrada en México: todas ellas datan de la segunda mitad del siglo XX; en su mayoría son de propiedad privada y recientemente fueron restauradas $y$ reubicadas en los tréboles viales de Insurgentes-Periférico y Viaducto-Periférico, gracias a un contrato de comodato entre los diferentes particulares y la colaboración entre una asociación civil y una organización no gubernamental.

\section{La gestación y consolidación del patrimonio cultural edificado y la sucesiva destrucción de este en la zona sur de la Ciudad de México (1900-1980)}

El primer objetivo particular es relacionar el proceso de reconocimiento y consolidación del patrimonio cultural edificado en México con la expansión física de la Ciudad de México hacia la zona sur, dentro de una temporalidad que abarca desde principios del siglo XX hasta la década de 1980. Con base en un enfoque histórico, se describen las in(acciones) particulares que gradualmente atraviesan los tres casos de análisis alrededor de su valoración (o no) como patrimonio cultural edificado en la región: la Zona Arqueológica de Cuicuilco, las esculturas monumentales de la XIX Olimpiada y la ex-fábrica de Peña Pobre.

De este modo, se establece una secuencia cronológica subdividida en tres etapas que obedecen a múltiples factores y acontecimientos político-económicos de México, entrelazándose en el devenir del tiempo y reflejando diferentes procesos en el área de estudio. La primera etapa de análisis abarca las décadas de 19001930, la segunda etapa de 1940-1970, y la tercera etapa se centra en la década de 1980. Con base en esta trayectoria histórica, la etapa de 1900-1930 se caracteriza por la construcción del proyecto de Estado-Nación en México. Esto trae consigo un doble producto en materia de patrimonio cultural hacia finales de la década de 1930: 1) el surgimiento de una institución cuyo pilar es la arqueología e historia de México, y 2) la cristalización de las sociedades prehispánicas como símbolo de identidad nacional en el marco de una apuesta por consolidar un proyecto de Estado-Nación, sentando las bases que, a la postre, darán forma a la institucionalización del patrimonio cultural edificado en apego a los valores estéticos, simbólicos, históricos y académicos (Bernal, 1979; Litvak y López, 2013; Lombardo, 2013; Lozoya, 2010).

A nivel local, durante la primera etapa inician las exploraciones en el asentamiento prehispánico de Cuicuilco entre 1924 y 1925 (Cummings, 1926; Haury, 1975, 2004), gracias al impulso que el Estado mexicano otorga a los estudios prehispánicos desde las postrimerías del gobierno de Porfirio Díaz hasta el de Lázaro Cárdenas. Esta etapa culmina hacia 1939, año en que se habilita la zona arqueológica para el acceso gratuito al público desde entonces. Así Cuicuilco se devela como el primer arquetipo de reconocimiento y valoración del patrimonio cultural edificado a nivel local entre las décadas de 19001930. Por un lado, gracias a su arquitectura monumental prehispánica entendida como símbolo fehaciente de un pasado glorioso, por el otro, debido a la institucionalización de la arqueología mexicana con motivo de la creación del Instituto Nacional de Antropología e Historia en 1939; todo ello en el marco de un Estado mexicano posrevolucionario que ensalza el nacionalismo. En las décadas posteriores, múltiples trabajos arqueológicos demostrarán las cualidades estructurales urbanas de Cuicuilco (Heizer \& Bennyhoff, 1958; Palerm y Wolf, 1972; Pérez, 2007; Rodríguez, 1994), encumbrándose como uno de los asentamientos con rasgos urbanos más antiguos de la Cuenca de México (Schávelzon, 1993), fortaleciendo su sentido de reconocimiento y preservación como patrimonio cultural edificado en la zona sur de la Ciudad de México.

La segunda etapa transcurre entre 1940-1970 y aparecen nuevos factores como la implementación del modelo de Industrialización por Sustitución de Importaciones [ISI] en México (Portes y Roberts, 2008) y su impacto en la expansión física de la Ciudad de México hacia el sur. En 1946 se crea el Instituto Nacional de Bellas Artes y Literatura [INBA], y tiempo después se oficializan los acuerdos internacionales que versan sobre la 
protección del patrimonio mundial, cultural y natural (UNESCO, 1972). A pesar de ello, entre los años de 1967 y 1968, en el clímax de la expansión de la Ciudad de México hacia el sur, se construyen grandes obras de infraestructura y equipamientos necesarios para la celebración de los Juegos Olímpicos dentro de los terrenos próximos a la Zona Arqueológica de Cuicuilco, principalmente la Villa Olímpica (Müller, 1990) (Figura 2).

Más aún, la conclusión de la Avenida Insurgentes hasta el entronque con la carretera Cuernavaca provoca la fragmentación del conjunto arquitectónico en dos partes. En la actualidad, la sección oriental comprende el polígono mayor abierto al público denominado Cuicuilco $A$, mientras que al costado oeste de la avenida se localizan los complejos B y C de Cuicuilco; el primero quedaría inmerso en las instalaciones de la Villa Olímpica, y el segundo sería destruido años más tarde para edificar el Corporativo Elektra (Pérez, Rangel y Sandoval, 2007; Rodríguez, 1994). Esto se aprecia como un primer momento de destrucción del patrimonio cultural edificado en la región, manifestándose como una contradicción hacia los fundamentos de protección y conservación del patrimonio cultural edificado instaurados en México desde el primer tercio del siglo $\mathrm{XX}$, en los cuales las sociedades prehispánicas se realzan como un símbolo de identidad nacional. Más aún, este proceso de cohesión identitaria basado en los valores simbólicos e históricos se ve bruscamente afectado por las decisiones políticas del gobierno local y federal que busca posicionar al país en el escenario internacional de la modernidad, fungiendo como anfitrión de la XIX Olimpiada en el año de 1968, pero olvidándose de los principios fundamentales del proyecto de Estado-Nación.

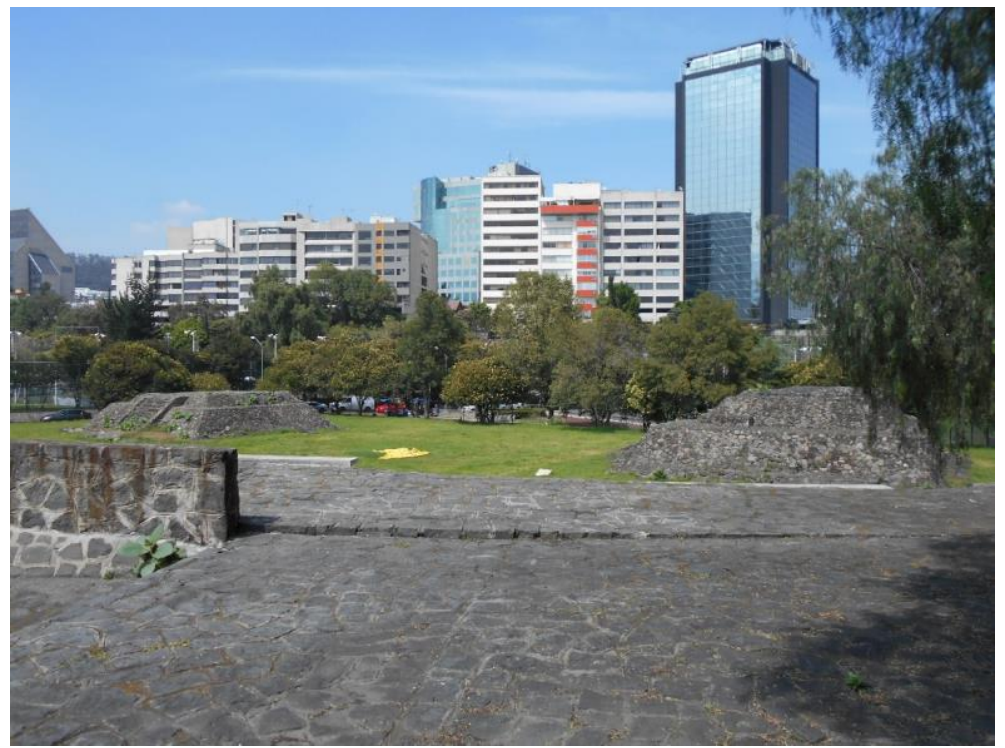

Figura 2. Complejo B de Cuicuilco dentro de las instalaciones de la Villa Olímpica: en primer plano se aprecian las estructuras prehispánicas VI y VIII; en segundo plano sobresale una línea de edificios ubicados en el corredor urbano Periférico Sur.

Fuente: Foto del autor.

A la par, en el marco de las celebraciones olímpicas de 1968, Pedro Ramírez Vázquez y Mathias Goeritz impulsan la construcción de esculturas monumentales como símbolo de hermandad entre México y el mundo (Wendl, 1998). Por invitación de ambos precursores, escultores nacionales y extranjeros plasman un total de 19 obras con diseños abstractos: 15 esculturas a lo largo del Anillo Periférico Boulevard Adolfo Ruiz Cortines, dos esculturas en la Avenida Insurgentes Sur, una escultura dentro de las instalaciones de la Villa Olímpica, y una escultura a la entrada del Embarcadero de Cuemanco Xochimilco, constituyendo así la denominada Ruta de la Amistad (Patronato Ruta de la Amistad, 2017). Gracias a esta iniciativa, las esculturas monumentales quedarían integradas como elementos artísticos y simbólicos de la imagen urbana desde su origen. A pesar de su naturaleza, es importante subrayar que las esculturas conmemorativas de la XIX Olimpiada no fueron reconocidas oficialmente ni valoradas como patrimonio 
cultural edificado por parte de las instituciones mexicanas, especialmente el INBA, durante todo el siglo XX.

La tercera etapa ocurre en la década de 1980. Su característica principal es la transición al modelo socioeconómico de Desarrollo orientado a las Exportaciones [EOD] de la mano con la implementación de políticas neoliberales en México. En la zona sur de la Ciudad de México acontecen nuevos casos aislados de destrucción del patrimonio cultural, los cuales se asocian, esta vez, con la edificación de grandes centros comerciales y centros médicos especializados sobre la Avenida Insurgentes Sur y el Anillo Periférico Boulevard Adolfo Ruiz Cortines (Marmolejo y Treviño, 2014). Como ya se discutió arriba, ambas vías gradualmente se convertirán en los nuevos ejes estructuradores de la Zona Metropolitana del Valle de México bajo la forma de corredores urbanos inclinados a satisfacer las demandas del sector terciario. En lo que concierne al patrimonio cultural edificado, la exfábrica de Peña Pobre, ubicada en los confines del centro histórico de Tlalpan, es catalogada como Monumento Histórico por el Instituto Nacional de Antropología e Historia por tratarse de un inmueble construido en el siglo XIX (INAH, 1988), cerrando de este modo un ciclo de políticas proteccionistas alrededor del patrimonio cultural edificado en la región de estudio.

\section{La reestructuración morfológica y funcional de Periférico Sur e Insurgentes Sur en corredores urbanos de carácter terciario (1980-2016)}

A manera de recapitulación histórica, las ciudades mexicanas atraviesan reconfiguraciones territoriales intrínsecamente relacionadas con la transición al modelo de Desarrollo orientado a las Exportaciones (EOD) ${ }^{1}$, gestándose, a partir de la década de 1980, una nueva lógica de distribución territorial en el caso de la Ciudad de México (Aguilar, 2003; Gobierno del Distrito Federal, 2000). En este sentido, trabajos recientes identifican la formación y consolidación de Periférico Sur e Insurgentes

\footnotetext{
${ }^{1} \mathrm{~A}$ grandes rasgos, el modelo de Desarrollo orientado a las Exportaciones [EOD] se integra de siete políticas principales siguiendo los trabajos de Portes (1997, p. 238) y Portes y Roberts (2008, p. 17):

1) la apertura unilateral del comercio exterior; 2) la extensa privatización de las empresas del Estado; 3) la desregularización de bienes, servicios y mercados laborales; 4) la liberalización de mercados de capital con privatización de los fondos de pensiones; 5)
}

Sur como dos de los principales corredores urbanos en la zona sur de la Ciudad de México desde la década de 1980 hasta el presente, caracterizados por una alta concentración del sector terciario y un patrón espacial de subcentros lineales continuos, aglutinando espacios con usos de suelo residenciales, corporativos y comerciales (Aguilar \& Hernández, 2015; Aguilar y Mateos, 2011; Pradilla, Márquez, León y Fonseca, 2008). De hecho, se revela que los principales motores en la dinámica territorial de los corredores urbanos Periférico Sur e Insurgentes Sur son:

"La euforia del capital inmobiliario derivada de una sobrestimación de la localización de nuevas oficinas corporativas a raíz de la entrada en vigor en 1994 del TLCAN, y la penetración del capital comercial trasnacional bajo la forma de filiales $y$ franquicias de cadenas extranjeras" (Pradilla y Pino, 2004, pp. 79-80).

Con base en el estado de la cuestión antes señalado, resulta conveniente dilucidar el carácter terciario del Anillo Periférico Boulevard Adolfo Ruiz Cortines y la Avenida Insurgentes Sur bajo la modalidad de nuevos ejes estructuradores de la ZMVM en la actualidad. Se parte de la premisa que, la realización de un análisis que ahonde en la categorización explícita del sector terciario a nivel espacial puede brindar soporte a la discusión del impacto de las políticas neoliberales en la dinámica territorial de esta región y, por ende, en el patrimonio cultural edificado allí presente.

En consecuencia, se plantea la siguiente metodología que conjuga un carácter cuantitativo y espacial. En primer lugar, a razón de categorizar el sector terciario, se conjuntan las variables de análisis de Peter Taylor (2004) y Peter Hall (2001) con dos tipos de servicios identificados dentro de la región, en aras de abrir el abanico de servicios avanzados. Por un lado, Taylor (2004) plantea la construcción de una tabla de servicios globales que denomina Matriz de Servicios, la cual sopesa en las

el ajuste fiscal basado en una reducción del gasto público; 6) la reestructuración de programas sociales estatales focalizados en esquemas compensatorios para los grupos más necesitados; 7) el fin de la política industrial y de cualquier otra forma de empresarialidad patrocinada por el Estado y la concentración en la gestión macroeconómica. 
variables de Publicidad, Banca-Finanzas, Contabilidad, Administración-Consultoría, Seguros y Jurídico. Por su parte, Hall (2001) establece una tipología relativa a la estructura policéntrica de las urbes, destacando los Centros de Educación Superior y Centros de Investigación Científica, al igual que los Complejos Deportivos. Finalmente, la presente investigación incluye como variables de análisis los centros de Medicina Especializada y Oficinas Centrales de Gobierno. Con ello se crea una tabla general que sirve de base en la identificación y cuantificación de servicios dentro los corredores urbanos Periférico Sur e Insurgentes Sur.

En segundo lugar, con apoyo del programa Google Maps, se procede a la identificación de servicios avanzados a lo largo de los corredores urbanos Periférico Sur e Insurgentes Sur, cuyos límites espaciales se mencionan al inicio del presente trabajo. De este modo, se retoma el Directorio Estadístico Nacional de Unidades Económicas que dispone el INEGI (2016) para la Ciudad de México hasta su última actualización como principal cuerpo de datos, el cual comprende nueve categorías de actividades económicas que se enlistan a continuación: a) Información en medios masivos; b) Servicios financieros y de seguros; c) Servicios profesionales, científicos y técnicos; d) Corporativos; e) Servicios de apoyo a los negocios; f) Servicios educativos; g) Servicios de salud; h) Servicios de esparcimiento, culturales y deportivos; i) Actividades legislativas, gubernamentales, de impartición de justicia y de organismos internacionales y extraterritoriales. Cabe mencionar que, uno de los principales criterios para la identificación y cuantificación de unidades económicas es su posición geográfica directa sobre ambos corredores urbanos.

Los resultados del análisis exhiben un total de 147 firmas de servicios avanzados en ambos corredores urbanos (Tabla 1). El corredor Insurgentes Sur despunta por un mayor número de empresas en los rubros de Jurídico [8 firmas], Educación Superior y Centros de Investigación Científica [8 firmas] y Oficinas Centrales de Gobierno [8 firmas]. En contraste, los rubros de Publicidad y Medicina Especializada son muy bajos en el corredor Insurgentes Sur, pues se reducen a una y tres firmas, respectivamente.

Tabla 1

Cuantificación de los diferentes tipos de firmas productoras de servicios avanzados en los corredores urbanos Periférico Sur e Insurgentes Sur

\section{Conteo de firmas/empresas productoras de servicios avanzados}

\begin{tabular}{|c|c|c|c|c|c|c|c|c|c|c|c|}
\hline Corredor Urbano & $\begin{array}{l}\frac{0}{0} \\
. \frac{\pi}{0} \\
. \frac{0}{0} \\
\frac{0}{2} \\
2\end{array}$ & 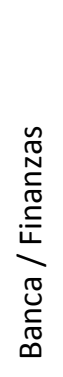 & $\begin{array}{l}\frac{d}{\pi} \\
\frac{\pi}{0} \\
\frac{0}{\overline{0}} \\
\frac{0}{\pi} \\
\tilde{0} \\
0 \\
0\end{array}$ & 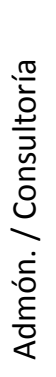 & 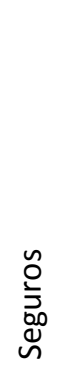 & 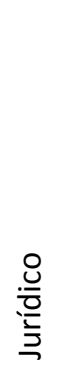 & 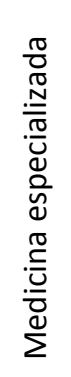 & 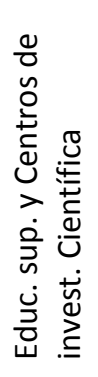 & 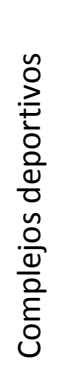 & 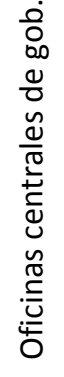 & $\begin{array}{l}\bar{\pi} \\
\stackrel{0}{\circ} \\
\end{array}$ \\
\hline Insurgentes Sur & 1 & 5 & 5 & 9 & 6 & 8 & 3 & 8 & 8 & 8 & 61 \\
\hline Periférico Sur & 9 & 5 & 5 & 18 & 9 & 3 & 14 & 7 & 8 & 7 & 86 \\
\hline Total & 10 & 11 & 10 & 27 & 15 & 11 & 17 & 15 & 16 & 15 & 147 \\
\hline
\end{tabular}

Fuente: Elaboración propia. 
En contraparte, el corredor Periférico Sur aventaja en el número de firmas de servicios relativos a los rubros de Publicidad [9 firmas], Administración-Consultoría [18 firmas], Seguros [9 firmas] y Medicina Especializada [14 firmas], mientras que el rubro de Jurídico es bajo con sólo tres firmas. De igual manera, la cuantificación evidencia tres rubros cuyo número es equivalente en ambos corredores urbanos; se trata de la Contabilidad [5 firmas], Banca-Finanzas [5 firmas], y Complejos Deportivos [8 firmas]. En términos porcentuales, el corredor Insurgentes Sur aglutina el $41,5 \%$ con un total de 61 firmas, mientras que el corredor Periférico Sur concentra el 58,5\% con 86 firmas.

Esta singular concentración de 147 firmas productoras de servicios avanzados, producto de la identificación inicial de unidades económicas contenidas en datos de INEGI (2016), la posterior ubicación espacial sobre los corredores urbanos Periférico Sur e Insurgentes Sur, y la ulterior categorización y sumatoria con base en las variables de Peter Taylor (2004), Peter Hall (2001) y dos variables propuestas en la presente investigación, brinda una idea acerca de la proliferación del sector terciario en cantidad y variabilidad en la actualidad. Si bien los datos analizados corresponden sólo al año 2016, permiten reconocer de forma explícita la predominancia de usos de suelo, complejos de edificaciones y comercios que se dedican al sector terciario en el área de estudio. Bajo la figura de plazas comerciales, edificios de corporativos en materia de publicidad, banca, finanzas, contabilidad, administración, consultoría, seguros y bufetes jurídicos, al igual que centros especializados de carácter médico, deportivo y educativo, todas estas nuevas formas urbanas adquieren sentido gracias al apoyo del Estado mexicano como el principal facilitador en el proceso de reconfiguración urbana, apegándose a la lógica de las políticas neoliberales en cuanto a la desregularización de mercados, bienes y servicios, aunado a los derechos de propiedad privada. Lo anterior pone en manifiesto la efectividad de los mecanismos ideológicos del propio sistema capitalista, favoreciendo los intereses del sector privado.
Las contradicciones en la preservación del patrimonio cultural edificado y su relación con la reconfiguración de Periférico Sur e Insurgentes Sur en corredores urbanos (presente)

Una vez analizado el proceso de reestructuración morfológica y funcional de Periférico Sur e Insurgentes Sur, en correlación con el predominio actual de edificaciones, comercios y usos de suelo inclinados al sector terciario, el tercer objetivo particular es analizar el impacto de ambos corredores urbanos en los tres casos de bienes patrimoniales citados anteriormente, a casi cuarenta años de haberse implementado las primeras políticas en México que favorecen la desregularización de bienes y servicios del Estado, los cambios al régimen de propiedad privada en áreas que originalmente pertenecen al Estado, en conjunto con los ajustes fiscales basados en la reducción del gasto público.

El caso de la Zona arqueológica de Cuicuilco. A grandes rasgos, se trata de un área de propiedad federal ubicada en la intersección de los corredores urbanos Periférico Sur e Insurgentes Sur. Se encuentra abierta al público de forma gratuita y se mantiene de un presupuesto federal bajo la custodia del INAH, por lo que la derrama económica del turismo cultural es nula. El primer objeto de estudio versa sobre la afluencia de público recurrente. Con base en el Reporte anual de estadística de visitas a museos y zonas arqueológicas del INAH (2017), la Zona arqueológica de Cuicuilco recibió un total de 80.726 visitantes en el año 2015 (Tabla 2). Los datos indican que los mayores porcentajes de visitantes se distribuyen entre los estudiantes de niveles básico [25,02\%] y superior [21,27\%], el público nacional en visita dominical [23,24\%] y el público nacional en visita regular [22,39\%]. En contraparte, los porcentajes más bajos corresponden a extranjeros [5\%], profesores [1,28\%], personas de la tercera edad [1,29\%] y personas con discapacidad [0,36\%]. Este conteo demuestra que en la Zona arqueológica de Cuicuilco perdura un flujo importante de visitantes al año que son principalmente estudiantes y público de origen nacional, cuya finalidad principal obedece a cuestiones educativas y de esparcimiento en similitud de proporciones. 
Tabla 2

Conteo de visitantes a la Zona arqueológica de Cuicuilco y museo de sitio en el año 2015

\begin{tabular}{|c|c|c|c|c|c|c|c|c|}
\hline \multicolumn{9}{|c|}{ Reporte anual de estadística de visitas a museos y zonas arqueológicas en 2015} \\
\hline Mes & 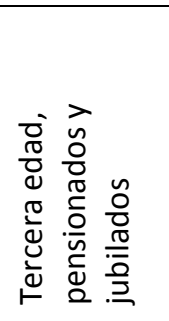 & 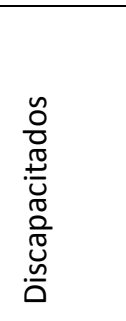 & 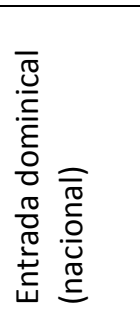 & 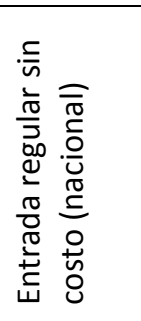 & 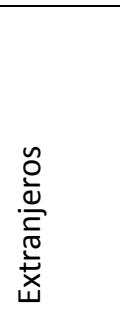 & 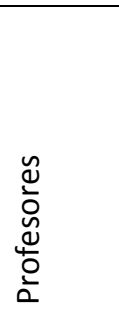 & 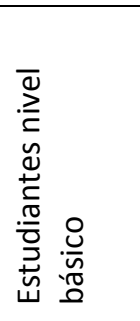 & 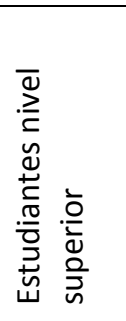 \\
\hline 1 & 46 & 5 & 1.330 & 1.109 & 252 & 104 & 1.740 & 578 \\
\hline 2 & 43 & 14 & 1.374 & 1.003 & 283 & 134 & 1.463 & 1.116 \\
\hline 3 & 183 & 18 & 1.936 & 3.465 & 280 & 172 & 2.737 & 1.291 \\
\hline 4 & 33 & 2 & 1.337 & 1.981 & 367 & 104 & 1.769 & 983 \\
\hline 5 & 102 & 6 & 1.166 & 1.859 & 378 & 82 & 937 & 1.077 \\
\hline 6 & 71 & 7 & 731 & 1.177 & 293 & 85 & 1.125 & 916 \\
\hline 7 & 81 & 0 & 1.150 & 556 & 470 & 30 & 1.225 & 885 \\
\hline 8 & 222 & 180 & 1.518 & 106 & 596 & 104 & 1.274 & 2.666 \\
\hline 9 & 80 & 14 & 3.146 & 623 & 296 & 42 & 2.042 & 2.075 \\
\hline 10 & 11 & 39 & 1.813 & 1.988 & 290 & 93 & 2.468 & 1.957 \\
\hline 11 & 77 & 5 & 2.296 & 2.463 & 285 & 77 & 2.449 & 1.884 \\
\hline 12 & 94 & 3 & 969 & 1.746 & 343 & 10 & 973 & 1.748 \\
\hline Total & 1043 & 293 & 18.766 & 18.076 & 4.133 & 1.037 & 20.202 & 17.176 \\
\hline$\%$ & 1,29 & 0,36 & 23,24 & 22,39 & 5,11 & 1,28 & 25,02 & 21,27 \\
\hline
\end{tabular}

Fuente: Elaboración propia basada en datos del INAH, 2017.

El segundo objeto de estudio surge de planteamientos anteriores en los que se afirma que los ajustes fiscales basados en la reducción del gasto público limitan al Estado mexicano como el principal sustento en materia de recuperación y preservación del patrimonio cultural edificado (Pradilla, 1996). La situación predominante en la Zona arqueológica de Cuicuilco no es la excepción. Tras haber realizado observación directa en un lapso de dos veces por mes entre enero y junio de 2016, se corrobora que los trabajos se reducen a mantenimiento menor de forma periódica en el complejo de Cuicuilco A [zona abierta al público], perdurando un estado de obsolescencia en buena parte de sus instalaciones y servicios. El problema se agudiza en el Complejo de Cuicuilco B [dentro de las instalaciones de la Villa Olímpica], el cual exhibe un alto grado de abandono de los edificios prehispánicos ante la falta de mantenimiento durante el mismo periodo de asistencia. De hecho, investigaciones previas evidenciaron esta constante hace más de diez años, elaborando entonces un diagnóstico general acerca de las condiciones de la Zona arqueológica de Cuicuilco (Peña, 2005).

Desde la perspectiva de este análisis, los limitados esfuerzos de investigación y preservación de la Zona arqueológica de Cuicuilco por parte del Estado mexicano son producto, sólo en parte, de los recortes al gasto público destinado al patrimonio cultural edificado. Por ejemplo, con base en el Presupuesto de Egresos de la Federación para el Ejercicio Fiscal aprobado por la Cámara de Diputados (Diario Oficial de la Federación, 2011-2017), al inicio del sexenio del Presidente Enrique Peña Nieto en 2012, el INAH obtuvo recursos específicos en apoyo a la investigación científica y la productividad a través del Programa de Ciencia, Tecnología e Innovación por 
212.000.000. ${ }^{2}$ Si bien en 2016 hubo un incremento de hasta el $27,1 \%$ en esta partida presupuestal con respecto al primer año de gobierno, al cierre de su administración se aprobaron MXN\$156.000.000, lo que representa una reducción del 73,57\% en seis años (Tabla 3 ). ${ }^{3}$

De igual modo, datos recientes indican que el presupuesto total asignado en 2017 al ramo de la Cultura fue de MXN\$12.428.000, en contraste con los MXN\$15.255.000 asignados en 2016, lo que representa una reducción del $21,1 \%$ con respecto al año anterior (Periódico El Universal, 2016). Otras fuentes hemerográficas apuntan un recorte que oscila entre 30 y $36 \%$ en 2017 , ya que incorporan otras variables como son los subsidios federales a las instituciones estatales, los programas de apoyo etiquetados a la infraestructura cultural, así como las actividades municipales y comunitarias (Garduño y Méndez, 2016; Leñero, 2016).

\section{Tabla 3}

Recursos asignados al INAH para llevar a cabo proyectos específicos durante el sexenio del presidente de México Enrique Peña Nieto (20122018).

\begin{tabular}{cc}
\hline Año & $\begin{array}{c}\text { Monto total (pesos } \\
\text { mexicanos) }\end{array}$ \\
\hline 2012 & 212.113 .516 \\
\hline 2013 & 209.402 .304 \\
\hline 2015 & 232.059 .647 \\
\hline 2016 & 250.093 .227 \\
\hline 2018 & 269.747 .245 \\
\hline
\end{tabular}

Fuente: Elaboración propia basada en el Presupuesto de Egresos de la Federación para el Ejercicio Fiscal 2012-2018).

\footnotetext{
2 En diciembre de 2015 ocurren cambios estructurales en la política cultural de México: se crea la Secretaría de Cultura y desaparece el Consejo Nacional para la Cultura y las Artes [CONACULTA], mientras que el INAH y el INBA pasan a depender de esta nueva Secretaría de Estado. Desde su creación, estas instituciones fueron organismos desconcentrados dependientes de la Secretaría de Educación Pública.
}

Es un hecho que el Estado limita su campo de intervención a acciones focalizadas de corta duración y alcance. No obstante, el fenómeno quizás va más allá de los ajustes fiscales basados en una reducción del gasto público como principal caso de política neoliberal. La otra parte podría atribuirse a la urgente necesidad de un replanteamiento en la estructura orgánica del INAH para afrontar, eficientemente, estas prácticas políticas económicas sin olvidarse de los fundamentos y valores originales que le otorgan virtudes al patrimonio cultural edificado de México; en esencia porque es la principal dependencia federal encargada de su investigación y conservación.

Por último, el tercer objeto de estudio es un factor que impacta en la falta de preservación de Cuicuilco y tiene relación directa con el auge de comercios y edificaciones dedicadas al sector terciario en las inmediaciones de su posición geográfica: la intersección de los corredores urbanos terciarios Periférico Sur e Insurgentes Sur. Vale recordar que, desde la década de los setenta, el "rápido desarrollo urbano" y "las grandes obras públicas y privadas" se consideran factores de riesgo para el patrimonio cultural y natural (UNESCO, 1972, Artículo 11). Bajo esta línea, la atención gira hacia el Centro Comercial Perisur y la Plaza Cuicuilco Inbursa, Centro Comercial y Cultural. Estas grandes plazas comerciales aledañas dieron gran impulso a la reestructuración morfológica y funcional de Periférico Sur e Insurgentes Sur y, en consecuencia, en el área circundante a la Zona arqueológica de Cuicuilco. Su proyección a convertirse en los principales hitos de los corredores urbanos más importantes en la zona sur de la Ciudad de México provocó "un alza en los precios del suelo, cambios en los usos del suelo, problemas de tráfico, saturación de estacionamientos, y severos cambios en la imagen urbana" (Gobierno del Distrito Federal, 2000, p.270). Así la demanda de terrenos aledaños a la Zona arqueológica de Cuicuilco pronto se materializó en la sede de corporativos y oficinas centrales de gobierno. Basta recordar que la demanda inmobiliaria en la zona provocó la destrucción total del Complejo de Cuicuilco C para la

\footnotetext{
${ }^{3}$ El uso de estos datos como soporte se debe a que están relacionados con el quehacer arqueológico particularmente, a diferencia del presupuesto total del INAH que engloba las partidas salariales, las prestaciones y demás gastos de la burocracia.
} 
construcción del Corporativo Elektra en la década de 1990 (Rodríguez, 1994).

A grandes rasgos, los intereses de la dinámica de acumulación de capital se mantienen, por ahora, al margen de los nulos ingresos del turismo cultural en la Zona arqueológica de Cuicuilco, predominando la afluencia de visitantes con fines educativos y de esparcimiento. Sin embargo, la demanda de terrenos para la construcción de nuevos desarrollos inmobiliarios se mantiene latente y en aumento. De tal suerte, el subrayar esta constante sirva para advertir de posibles nuevas afectaciones al patrimonio cultural edificado en esta zona producto de las políticas neoliberales, las cuales resultarían en la desregularización de bienes y servicios del Estado, así como el otorgamiento de derechos de propiedad privada, o bien concesiones en áreas que hasta la fecha son de propiedad federal; en otros términos, cambios de uso de suelo en favor del sector terciario y la inminente destrucción de la Zona arqueológica de Cuicuilco y su área natural de conservación.

El caso de la ex-fábrica de Peña Pobre. A grandes rasgos, la historia de este inmueble se remonta a mediados del siglo XIX; su construcción en la periferia de la Villa de Tlalpan tuvo la finalidad de albergar una de las industrias papeleras más importantes en México (Lenz, 1957). Su cierre definitivo en 1987 es producto de factores tales como los problemas financieros de la compañía y la imposición de sanciones gubernamentales a causa del deterioro ambiental (Departamento del Distrito Federal, 1987 - 1988; Muñoz, 2011). En la etapa inmediata al cierre, el predio de la ex-fábrica en general, incluyendo el casco, la capilla y el espacio circundante no construido, atraviesan por un proceso de obsolescencia funcional y económica (Rojas, Rodríguez y Wegelin, 2004). En otros términos, el antiguo edificio se posiciona en el umbral de la obsolescencia funcional porque deja de tener un uso industrial. Igualmente, su ubicación en la Avenida Insurgentes Sur le otorga una alta plusvalía al predio, misma que para entonces se halla en proceso de consolidación como corredor urbano. Lo anterior se vuelve un factor que agudiza el proceso de obsolescencia económica del inmueble en el supuesto de haber mantenido su función original como fábrica.

Bajo esta línea se revelan importantes factores coyunturales en la zona sur de la Ciudad de México hacia finales de los ochenta: 1) la acelerada transformación de la avenida Insurgentes Sur en corredor urbano terciario ocurre durante la transición al modelo de Desarrollo orientado a las Exportaciones en México; 2) el cierre definitivo de la fábrica en relación con la industria papelera; 3) la prevalencia de un edificio catalogado como Monumento Histórico por las instituciones mexicanas ante un inminente proceso de obsolescencia física, funcional y económica; 4) las limitaciones del Estado mexicano en materia de adquisición, recuperación y preservación del patrimonio cultural edificado derivadas de la implementación de políticas que apuntan hacia los ajustes fiscales basados en la reducción del gasto público.

Al poco tiempo el inmueble es adquirido por el sector privado; el antiguo casco se remodela y transforma en la Plaza Cuicuilco Inbursa para el año de 1997 (Figura 3). Ahora bien, en trabajos anteriores la ex-fábrica de Peña Pobre se destaca como "un parteaguas en la reconversión de antiguas fábricas en centros urbanos de comercio, entretenimiento y cultura, generando nuevos puntos de encuentro y socialización para la población" (Sordo, 2013, pp. 130-131). Esta afirmación es cuestionable por lo siguiente. Desde la presente postura, su refuncionalización se convierte en un caso de destrucción creativa del patrimonio cultural edificado acorde a la lógica de la reproducción capitalista (Delgadillo, 2014; Harvey, 2003) a partir de la construcción de nuevos espacios privados con uso público. En otros términos, la reconversión del edificio histórico con fines socioeconómicos por parte del sector privado revela la destrucción (in)voluntaria del patrimonio cultural con fines de re(creación), siguiendo las premisas de Choay (2006). 


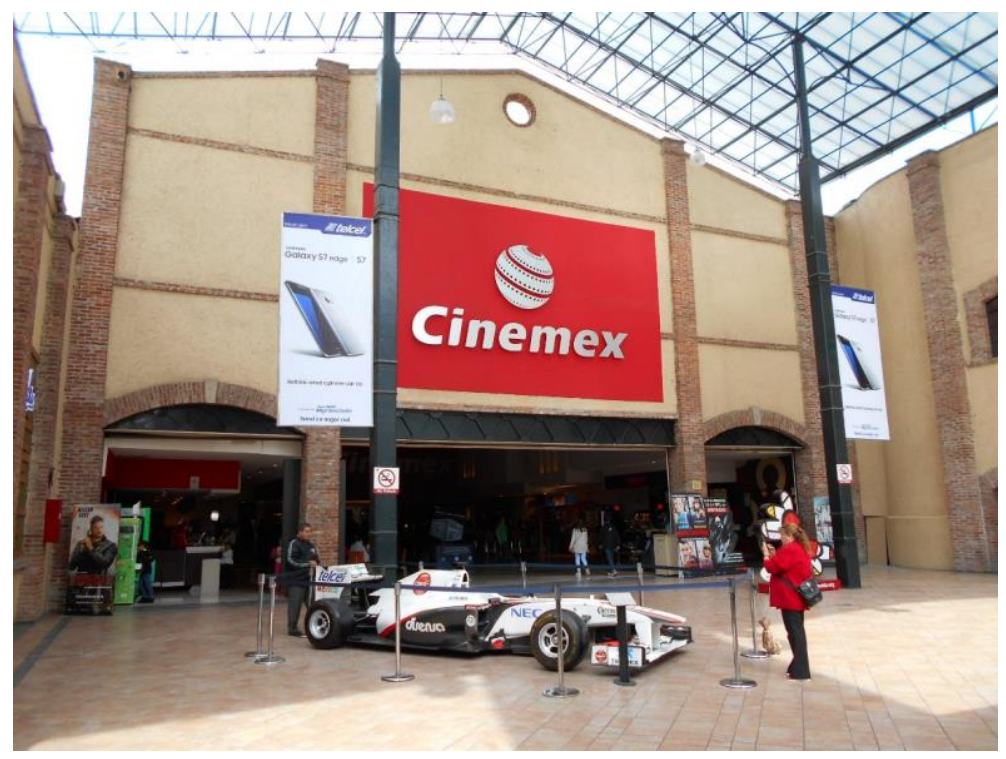

Figura 3. Estado actual de la antigua fábrica de Peña Pobre tras su reconversión como plaza comercial.

Fuente: Foto del autor.

Cabe señalar que, en el contexto internacional, también hay casos de reconversión de antiguos espacios y edificios que en el pasado tuvieron un uso industrial y en la actualidad disfrutan de un uso comercial dentro de las ciudades. Lo anterior ha resultado en diversas críticas hacia este tipo de intervenciones. Por ejemplo, destaca la Hay's Galleria en el distrito de Southwark, Londres, por los cambios en los roles de los sectores público y privado en el rediseño de la estructura urbana (Marmot y Worthington, 1986). Otro caso es el High Line Park en el distrito de Manhattan, Nueva York, en donde las críticas versan hacia los fines de lucro del sector privado en estos espacios reconvertidos (Loughran, 2017), o bien se habla de cuestiones de desigualdad en cuanto a la inversión de recursos para otros parques dentro de áreas más pobres de la ciudad (Morenas, 2012).

En el caso de la ex-fábrica de Peña Pobre hay una lógica particular que subyace en la restauración de este inmueble histórico por parte del sector privado. En concreto, no se busca reiterar o apuntalar las características que hacen única y diferente una cultura frente a otra; tampoco se pretende consolidar la memoria colectiva de los residentes de la ciudad (Rossi, 1981), ni la identidad de los habitantes de la zona sur de la Ciudad de México, a razón de los motores que pueden impulsar la conservación de inmuebles. Caso contrario, la reconversión tiene como finalidad entrar a la esfera de acumulación de capital, pues al término del proyecto, el antiguo casco disfruta de un valor de uso directo mediante su rehabilitación para fines comerciales (Rojas et al., 2004), mientras que los grandes espacios aledaños sacan provecho de un valor de uso indirecto mediante la construcción de edificios de corporativos, un centro de entretenimiento para niños, locales comerciales, restaurantes, entre otros.

Más aún, detrás de la reconversión del inmueble histórico para su ulterior promoción como Centro Comercial y Cultural, se afianza la potencialización de la cultura del consumo, aludiendo a un fenómeno que permea en la sociedad contemporánea y deriva en alteraciones a "las formas de vivir en su conjunto, marcan el estatus y el rango, las identidades de clase, edad, género y también su metamorfosis, migraciones $y$ mestizajes" (Zamora, 2007, p. 514). En consecuencia, no sólo se desvirtúa el uso y el aprovechamiento de los bienes culturales (Salas, 2007), sino que incluso se anulan los juicios originales bajo los cuales se consolida la noción de patrimonio cultural edificado, desvaneciendo los motores identitarios y la memoria colectiva que impulsan la conservación de este.

Por ende, la presente investigación coincide con la postura de Emilio Pradilla et al. (2008), quienes rechazan la supuesta función de las plazas comerciales en nuevas centralidades de corredores urbanos, al ser catalogados como "nuevos espacios públicos" en algunos trabajos (por 
ejemplo, Paquette, 2007); básicamente porque estos espacios construidos en la etapa del modelo socioeconómico EOD carecen de una "libre apropiación por los ciudadanos, de ámbitos verdaderamente públicos" (Pradilla et al., 2008, p.51), valorada ésta como el componente primordial de la vida urbana colectiva que da origen a los subcentros. A ello se suman ciertos rasgos develados en las plazas comerciales detrás de su etiqueta de nuevos espacios públicos, principalmente la proliferación de un marcador social que genera una mayor estratificación y polaridad entre los grupos que ahí acuden, aunado al hecho que, si bien se les otorga un carácter recreativo y activo, la convivencia y comportamiento es limitada e incluso regulada (Filipe, 2014). Por lo anterior, en las plazas comerciales, incluyendo la que hoy alberga la ex-fábrica de Peña Pobre, difícilmente se puede hacer referencia a ellas como espacios socialmente homogéneos donde se permita la libre reproducción de la vida social.

\section{El caso de las esculturas conmemorativas de la XIX} Olimpiada celebrada en México. En 1994 se crea un fideicomiso integrado por embajadas de diversos países, el sector público y algunas instituciones privadas cuya meta es la recuperación y preservación constante de las esculturas bajo la figura de Patronato Ruta de la Amistad A.C. (2017). Este esfuerzo se debe a la situación de abandono, la negligencia, el grave estado de conservación y la subutilización de espacios aledaños a las esculturas en pro de actividades culturales, que prevaleció durante décadas bajo su condición de propiedad privada (Amador, 2011; Terrazas, 1996; Ventura, 2011).

Dicha problemática se mantuvo constante hasta el 2011, año en que las esculturas son incluidas en la lista de monumentos en riesgo por parte de la World Monuments Fund (2017). Lo anterior es básicamente un inventario elaborado por esta ONG donde se exponen los bienes patrimoniales a nivel internacional que requieren de atención inmediata por el alto riesgo de destrucción. Este suceso se vuelve coyuntural en la aprobación de un proyecto que, a través del Patronato, se buscaba la restauración y reubicación de las esculturas, pues anteriormente el gobierno del Distrito Federal había anunciado el otorgamiento de una concesión a una empresa transnacional para la construcción de la Autopista Urbana Sur sobre un tramo del Anillo Periférico (Ventura, 2011). Gracias a la participación conjunta del Patronato y la ONG, en el año 2013 se logra la celebración de un contrato de comodato con los propietarios de las esculturas para su ulterior restauración y reubicación sobre los tréboles de Insurgentes-Periférico y ViaductoPeriférico (Figura 4), evitando su destrucción inminente a causa de la construcción de nuevas grandes obras de infraestructura vial en la zona sur de la Ciudad de México.

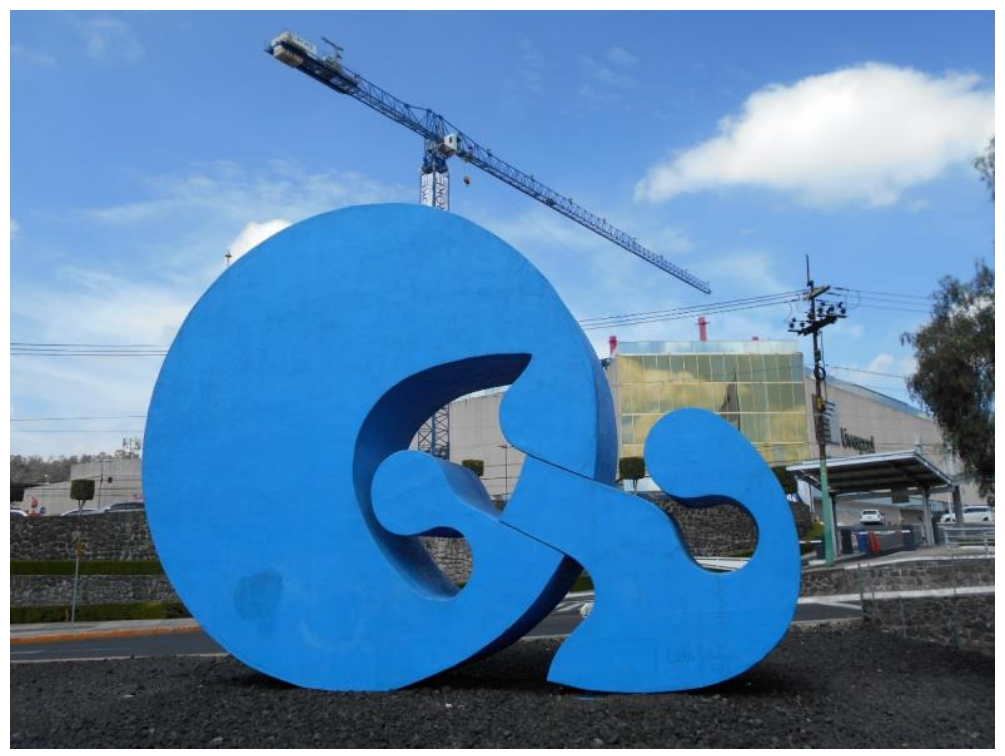

Figura 4. Escultura Estación 2-El Ancla de Willi Gutmann tras haber sido restaurada y reubicada en el trébol de Insurgentes Sur y Periférico Sur; en segundo plano se halla el Centro Comercial Perisur.

Fuente: Foto del autor. 
Las esculturas conmemorativas de la XIX Olimpiada también son una muestra de las contradicciones hacia los fundamentos y acciones de preservación del patrimonio cultural edificado en México. La razón es la siguiente. Hasta el año 2011, ninguna de las esculturas había sido reconocida oficialmente como Monumento Artístico por el Instituto Nacional de Bellas Artes, a pesar de estar marcadas por un hito en la historia del México contemporáneo, de su relevancia artística como nuevos elementos integrados a la imagen urbana, y del significado simbólico de hermandad entre las naciones expresado en la Ruta de la Amistad.

De acuerdo con los datos disponibles, al término de los Juegos Olímpicos de 1968, las esculturas fueron vendidas a particulares como parte de los terrenos, quedando sujetas a la voluntad o (des)interés de los propietarios (Amador, 2011). Esto demuestra que el hecho de que un monumento con ciertos valores históricos, simbólicos o artísticos se encuentre bajo el régimen de propiedad privada, no necesariamente garantiza acciones inherentes al reconocimiento, valoración y, por tanto, la destinación de recursos para la preservación de este. En resumen, el propósito de analizar detenidamente el caso de las esculturas conmemorativas de la XIX Olimpiada es advertir en lo siguiente. En una época de cambios en las políticas que favorecen la privatización de bienes del Estado, por un lado, y la delegación de responsabilidades por parte de este último a particulares, por el otro, no necesariamente es una decisión que garantice resultados prolíficos en el caso del patrimonio cultural edificado.

\section{Conclusiones}

Se confirma la hipótesis presentada al inicio de la investigación mediante la puesta en evidencia del fenómeno subyacente en la Zona arqueológica de Cuicuilco, la ex-fábrica de Peña Pobre y las esculturas conmemorativas de la XIX Olimpiada. De tal modo, las diferentes trayectorias de esta tercia de bienes patrimoniales están intrínsecamente relacionadas con la implementación de políticas neoliberales inclinadas hacia los ajustes fiscales basados en la reducción del gasto público, la desregularización de bienes y servicios del Estado, al igual que el otorgamiento de derechos de propiedad privada en áreas que originalmente son de propiedad federal, convirtiéndose en la constante que origina las contradicciones asociadas a las (in)acciones y los (des)intereses de diversos actores públicos y privados, mismas que se contraponen a los valores históricos, estéticos, simbólicos, sociales y académicos bajo los cuales se funde el concepto original de patrimonio cultural edificado en México.

El impacto de estas políticas en la dinámica territorial de la zona sur de la Ciudad de México actualmente se materializa en la concentración de usos de suelo y edificaciones que se dedican al sector terciario en los corredores urbanos Periférico Sur e Insurgentes Sur, incluyendo comercios, edificios de corporativos, complejos de carácter médico, deportivo y educativo. Esta afirmación se soporta a través de un conteo de 147 empresas/firmas productoras de servicios avanzados en esta región de estudio, los cuales se identifican gracias a un análisis espacial en cada corredor urbano que sopesa en diez variables. Por lo anterior, se sostiene que estas nuevas formas urbanas que predominan en la actualidad derivan de las políticas que promueven la desregularización de mercados, bienes y servicios y el otorgamiento de derechos de propiedad privada, especialmente, favoreciendo los intereses del sector privado.

Ahora bien, como primer caso de análisis, la Zona arqueológica de Cuicuilco evidencia el predominio anual de visitantes con fines educativos y de esparcimiento, manteniendo los intereses de la dinámica de acumulación de capital al margen hasta hoy. Esta afirmación se basa en un conteo anual que supera los 80 mil visitantes en 2015, de los cuales el $90 \%$ son estudiantes de niveles básico y superior, así como público de origen nacional. Ahora bien, la falta de mantenimiento y preservación en sus instalaciones y edificios prehispánicos se demuestra a partir de observación directa periódica durante el primer semestre de 2016. Por una parte, esto tiene relación directa con los recortes al gasto público destinado al patrimonio cultural en el marco de la implementación de las políticas neoliberales, mismo que en época reciente se ha visto afectado en un $30 \%$ menos. Por otra parte, se plantea que este fenómeno se deba también a la inminente necesidad de replantear la estructura orgánica del INAH, a razón de contraponerse a las prácticas de las políticas neoliberales sin desatender los fundamentos y valores originales que le otorgan reconocimiento al patrimonio cultural edificado de México, en esencia 
porque es la principal dependencia federal encargada de la investigación científica y la conservación de este.

En lo que concierne al segundo caso de análisis, los argumentos y datos expuestos alrededor de la ex-fábrica de Peña Pobre bajo su nueva faceta de plaza comercial en el corredor urbano Insurgentes Sur demuestran lo siguiente. En primer lugar, el edificio histórico y su terreno circundante atraviesan por un proceso de obsolescencia funcional y económica en el lapso inmediato al cierre de la fábrica; se abandona el uso industrial, a la vez que la función original del edificio y su terreno circundante dejar de ser rentables por la alta plusvalía de la zona, recordando que la Avenida Insurgentes Sur se hallaba, entonces, en pleno proceso de consolidación como corredor urbano. Segundo, la posterior reconversión de este edificio del siglo XIX en una plaza comercial se traduce en un espacio privado con uso público acorde a la lógica de la reproducción capitalista que sólo exterioriza una escenografía vintage; empero, el valor histórico que le otorga sentido como patrimonio cultural edificado, tanto como las cuestiones identitarias y la memoria colectiva que impulsan su conservación, todo ello se relega a segundo término. Tercero, los juicios originales que impulsan y cristalizan la noción de patrimonio cultural edificado en las leyes nacionales y los tratados internacionales se doblegan ante la cultura del consumo; la plaza comercial sólo se promueve y beneficia de la etiqueta cultural, pues en la práctica su finalidad es la acumulación de capital privado. Cuarto, se argumenta que la plaza comercial que hoy alberga la ex-fábrica de Peña
Pobre no puede considerarse una nueva centralidad o subcentro del corredor urbano Insurgentes Sur, básicamente porque no es un espacio socialmente homogéneo donde se permita la libre reproducción de la vida social.

Por último, el hecho que las esculturas conmemorativas de la XIX Olimpiada hayan carecido de reconocimiento y protección oficial por parte del INBA durante décadas revela una contradicción hacia los fundamentos y valores originales que dan sentido a las acciones de conservación del patrimonio cultural edificado en México. Así, las esculturas prevalecieron ante la indiferencia de los propietarios bajo condiciones de abandono y deterioro por mucho tiempo (Terrazas, 1996; Ventura, 2011), toda vez que fue necesaria la participación conjunta de asociaciones civiles y organizaciones no gubernamentales para lograr su rescate, restauración y reubicación definitiva sobre los tréboles viales de Insurgentes-Periférico y Viaducto-Periférico (Amador, 2011, 2013). Por ende, sirva este caso de análisis para rechazar afirmaciones a priori que sostengan que la privatización de bienes patrimoniales [reconocidos de antemano por su valor histórico, artístico, social, espiritual o simbólico] genera automáticamente acciones de valoración y ulterior destinación de recursos para la conservación de este. La delegación a particulares de acciones que originalmente son responsabilidad del Estado mexicano no necesariamente garantiza resultados positivos en el caso del patrimonio cultural edificado [B] 


\section{Referencias}

Aguilar, A. (2003). La megaurbanización en la Región Centro de México. Hacia un modelo de configuración territorial. En A. Aguilar (coord.), Urbanización, cambio tecnológico y costo social. El caso de la Región Centro, México (pp. 19-71). México: Universidad Nacional Autónoma de México.

Aguilar, A., \& J. Hernández (2015). Metropolitan Change and Uneven Distribution of Urban Sub-Centres in Mexico City, 1989-2009. Bulletin of Latin American Research, 1-19.

Aguilar, A. y Mateos, P. (2011). Diferenciación sociodemográfica del espacio urbano de la Ciudad de México. EURE, 37(110), 5-30. https://doi.org/10.4067/s0250$\underline{71612011000100001}$

Amador, J. (2011). Nuevo destino para la Ruta de la Amistad. Revista Proceso, México. Recuperado de http://www.proceso.com.mx/284841/nuevodestino-para-la-ruta-de-la-amistad

Amador, J. (2013). Reubicación de la Ruta de la Amistad. Revista Proceso, México. Recuperado de http://hemeroteca.proceso.com.mx/?p=341255

Bernal, I. (1979). Historia de la arqueología en México. México: Porrúa.

Borja, J. (2007). Revolución y contrarrevolución en la ciudad global. EURE, 33(100), 35-50. http://www.eure.cl/index.php/eure/article/view/13 $\underline{68}$

Carrión, F. (2012). Dime quién financia el centro histórico y te diré qué centro histórico es. En A. Ziccardi (Ed.), Ciudades del 2010: entre la sociedad del conocimiento y la desigualdad social (pp. 517551). México: Universidad Nacional Autónoma de México.

Choay, F. (2006). Pour une Anthropologie de L'Espace. Francia : Editions Du Seuil.

Cummings, B. (1926). Cuicuilco and the Archaic Culture of Mexico. The Scientific Monthly, 23(4), 289-304.
Delgadillo, V. (2014) Destrucción y conservación del patrimonio urbano. En Anuario de Investigación del Posgrado en Urbanismo, 1(0), 131-149).

Departamento del Distrito Federal (1987-1988). Programa General de Desarrollo Urbano del Distrito Federal. Dirección General de Reordenación Urbana y Protección Ecológica-DDF, México.

Diario Oficial de la Federación (2011-2017). Presupuesto de Egresos de la Federación para el Ejercicio Fiscal 2012 a 2018.

Filipe, C. (2014). La construcción, significado y prácticas del espacio público en la ciudad de Cuernavaca. En: Anuario de Investigación del Posgrado en Urbanismo, 1(0), (pp. 19-31), México: Universidad Nacional Autónoma de México.

Garduño, R. y Méndez, E. (2016). "Brutal” recorte a la cultura: comisión legislativa del ramo. Periódico La Jornada, México. https://www.jornada.com.mx /2016/09/14/politica/003n1pol\#

Gobierno del Distrito Federal (2000). La Ciudad de México hoy. Bases para un diagnóstico. Fideicomiso de Estudios Estratégicos sobre la Ciudad de México.

Hall, P. (2001). Global City-regions in the 21st Century. En A.J. Scott (ed.), Global City-Regions: Trends, Theory, Policy (pp. 59-77). New York: Oxford University Press.

Harvey, D. (2003). Espacios de esperanza. Madrid: Akal.

Harvey, D. (2007). Neoliberalism as Creative Destruction. The ANNALS of the American Academy of Political and Social Science, 610(1), 21-44.

Haury, E. (1975). Cuicuilco in Retrospect. Kiva, 41(2), 195-200.

Haury, E. (2004). Cuicuilco Diary: June 11-September 12, 1925. Journal of the Southwest, 46(1),55-91.

Heizer, R. \& Bennyhoff, J. (1958). Archaeological Investigation of Cuicuilco, Valley of Mexico, 1957. Science, New Series, 127(3292), 232-233.

Instituto Nacional de Antropología e Historia (1988). Catálogo Nacional. Monumentos Históricos Inmuebles. Tlalpan, D.F., México. 
Instituto Nacional de Antropología e Historia (2017). Reporte anual de estadística de visitas a museos y Zonas Arqueológicas en 2015. Recuperado de http://www.estadisticas.inah.gob. $\mathrm{mx} /$

Instituto Nacional de Estadística, Geografía e Informática [INEGI] (2015). Conjunto de datos vectoriales de la carta topográfica, escala 1:50 000, serie III, Ciudad de México, clave E14A39. Recuperado de http://www.beta.inegi.org.mx/app/mapas/?t=0150 001000000000

Instituto Nacional de Estadística, Geografía e Informática [INEGI] (2016). Directorio Estadístico Nacional de Unidades Económicas. Recuperado de http://www.beta.inegi.org.mx/app/mapa/denue/

Lenz, H. (1957). Loreto. Historia y evolución de una fábrica de papel. México: Fábricas de Papel Loreto y Peña Pobre, S.A.

Leñero, E. (2016). El desastre cultural del presupuesto. Revista Proceso, México. Recuperado de https://www.proceso.com.mx/464589/desastrecultural-del-presupuesto

Litvak, J., y López, S. (2013). El patrimonio arqueológico. Conceptos y usos. En E. Florescano (coord.), EI patrimonio nacional de México, Tomo II (pp. 172197). México: Consejo Nacional para la Cultura y las Artes/Fondo de Cultura Económica.

Lombardo, S. (2013). El patrimonio arquitectónico y urbano (de 1521 a 1900). En E. Florescano (coord.), El patrimonio nacional de México, Tomo II (pp. 198240). México: Consejo Nacional para la Cultura y las Artes/Fondo de Cultura Económica.

Loughran, K. (2017). Parks for Profit: Public Space and Inequality in New York City. En C. Lindner y B. Rosa (Eds.), Deconstructing the High Line: Postindustrial Urbanism and the Rise of the Elevated Park (pp. 6172). New Brunswick: Rutgers University Press.

Lozoya, J. (2010). Ciudades Sitiadas. Cien años a través de una metáfora arquitectónica. México: Tusquets Editores.

Marmolejo, E., y Treviño, M. (2014). Los pioneros en la investigación arqueológica de Coyoacán. Arqueología Mexicana, 22(129), 34-37.
Marmot A., y Worthington, J. (1986). Great Fire to Big Bang: Private and Public Designs on the City of London. Built Environment, 12(4):216-233.

Morenas, L. (2012). Critiquing Landscape Urbanism: A View on New York's High Line. Economic and Political Weekly, 47(7):19-22.

Muñoz, M. (2011). La vivienda obrera de la fábrica de papel Loreto: reflexiones para su apreciación y rescate (Tesis de Maestría). Escuela Nacional de Conservación, Restauración y Museografía "Manuel del Castillo Negrete", México.

Müller, F. (1990). La cerámica de Cuicuilco B: Un rescate arqueológico. México: Instituto Nacional de Antropología e Historia.

Palerm, A., y Wolf, E. (1972). Agricultura y civilización en Mesoamérica. México: Secretaría de Educación Pública.

Paquette, C. (2007). Comercio y planificación urbana. Las nuevas grandes centralidades comerciales en los planes de desarrollo urbano de la ciudad de México. TRACE, 51, 44-55.

Patronato Ruta de la Amistad A.C. (2017). Historia. 17 Recuperado de http://www.mexico68.org/es/

Peña, S. (2005). Plan de manejo para la zona arqueológica de Cuicuilco. Una herramienta para su conservación integral [Tesis de Licenciatura]. Escuela Nacional de Antropología e Historia, México.

Pérez, M. (2007). Preclásico Tardío (400 a.C. - 200 d.C.) Las primeras ciudades. Arqueología Mexicana, 86, 40-43.

Pérez, M., Rangel, F. y Sandoval, Z. (2007). Cuicuilco. Ciudad de México. México: Instituto Nacional de Antropología e Historia.

Periódico El Universal (11 de noviembre de 2016). Reducen presupuesto en Cultura para 2017. México. Recuperado de: http://www.eluniversal.com.mx/ articulo/cultura/2016/11/11/reducen-presupuestoen-cultura-para-2017

Portes, A. (1997). Neoliberalism and the Sociology of Development: Emerging Trends and Unanticipated Facts. Population and Development Review, 23: 229259. 
Portes, A. y Roberts, B. (2008). La ciudad bajo el libre mercado. La urbanización en América Latina durante los años del experimento neoliberal. En Portes, Roberts y Grimson (coords.), Ciudades Latinoamericanas. Un análisis comparativo en el umbral del nuevo siglo (pp. 13-59). México: Universidad Autónoma de Zacatecas/Miguel Ángel Porrúa.

Pradilla, E. (1996). Lo privado y lo público en el patrimonio del siglo XX en América Latina. En $\mathrm{M}$. Rodríguez Viqueira, y E. Pradilla Cobos (eds.), Memorias. Seminario sobre la conservación del patrimonio del siglo XX (pp. 75-78). México: Consejo Internacional de Monumentos y Sitios/Universidad Autónoma Metropolitana.

Pradilla, E., y Pino, R. (2004). Ciudad de México: de la centralidad a la red de corredores urbanos. Anuario de espacios urbanos, 2004:71-96. México: Universidad Autónoma Metropolitana-Azcapotzalco.

Pradilla, E., Márquez, L., Carreón, S. y Fonseca, E. (2008). Centros comerciales, privatización y tercerización de lo público. Ciudades, 79:44-53. Puebla: Red Nacional de Investigación Urbana.

Rodríguez, E. (1994). Cuicuilco C. Un rescate arqueológico [Tesis de Licenciatura]. Escuela Nacional de Antropología e Historia, México.

Rojas, E., Rodríguez, E. y Wegelin, E. (2004). Volver al centro, la recuperación de áreas urbanas centrales. Washington, D.C.: Banco Interamericano de Desarrollo.

Rossi, A. (1981). La arquitectura de la ciudad. Barcelona: Gustavo Gili.

Salas, R. (2007). La naturaleza de la gestión cultural. La valorización económica en México. Revista DADU, 2(1):45-50.

Schávelzon, D. (1993). La pirámide de Cuicuilco. México: Fondo de Cultura Económica.
Smith, N. (1984). Uneven Development: Nature, Capital, and the Production of Space. Oxford: Basil Blackwell.

Sordo, J. (2013). Antes y después de los Centros Comerciales. Real Estate Market \& Lifestyle, 92, 3843.

http://www.realestatemarket.com.mx/articulos/me rcado-inmobiliario/comercial/13038-antesydespues-de-los-centros-comerciales

Taylor, P. (2004). World City Network. A Global Urban Analysis. Londres: Routledge.

Terrazas, A. (31 de Agosto de 1996). La Ruta de la Amistad, a merced de los dueños de los terrenos: destruyen el basamento de la escultura de Australia. Proceso. Recuperado de http://www.proceso.com.mx/173119/la-ruta-de-laamistad-a-merced-de-los-duenos-de-los-terrenosdestruyen-el-basamento-de-la-escultura-deaustralia

United Nations Educational, Scientific and Cultural Organization [UNESCO] (1972). Convención sobre la protección del patrimonio mundial, cultural y natural. Conferencia general presentada en la 170 reunión de la UNESCO. París, Francia.

Ventura, A. (2011). Alerta, monumentos en riesgo. EI Universal, México. http://archivo.eluniversal.com. $\mathrm{mx} /$ cultura/66618.html

Wendl, K. (1998). The Route of Friendship: A Cultural/Artistic Event of the Games of the XIX Olympiad in Mexico City - 1968. OLYMPIKA: The International Journal of Olympic Studies, VII, 113134.

World Monuments Fund (2017). Ruta de la Amistad. A Closer Look. Recuperado de https://www.wmf.org/ project/ruta-de-la-amistad

Zamora, J. (2007). La cultura del consumo. Realidad: Revista de Ciencias Sociales y Humanidades, 114, 513-553. https://doi.org/10.5377/realidad.v0i114.5 $\underline{142}$ 